\title{
Effects of Consumption of Rooibos (Aspalathus linearis) and a Rooibos-Derived Commercial Supplement on Hepatic Tissue Injury by tert-Butyl Hydroperoxide in Wistar Rats
}

\author{
B. D. Canda, O. O. Oguntibeju, and J. L. Marnewick \\ Oxidative Stress Research Centre, Department of Biomedical Sciences, Faculty of Health and Wellness Sciences, Cape Peninsula \\ University of Technology, P.O. Box 1906, Bellville 7535, South Africa
}

Correspondence should be addressed to J. L. Marnewick; marnewickj@cput.ac.za

Received 8 October 2013; Revised 25 January 2014; Accepted 28 January 2014; Published 11 March 2014

Academic Editor: Cinzia Signorini

Copyright (C) 2014 B. D. Canda et al. This is an open access article distributed under the Creative Commons Attribution License, which permits unrestricted use, distribution, and reproduction in any medium, provided the original work is properly cited.

\begin{abstract}
This study investigated the antioxidative effect of rooibos herbal tea and a rooibos-derived commercial supplement on tert-butyl hydroperoxide- ( $t$-BHP-) induced oxidative stress in the liver. Forty male Wistar rats consumed fermented rooibos, unfermented rooibos, a rooibos-derived commercial supplement, or water for 10 weeks, while oxidative stress was induced during the last 2 weeks via intraperitoneal injection of $30 \mu$ mole of $t$-BHP per $100 \mathrm{~g}$ body weight. None of the beverages impaired the body weight gain of the respective animals. Rats consuming the rooibos-derived commercial supplement had the highest $(P<0.05)$ daily total polyphenol intake $(169 \mathrm{mg} /$ day) followed by rats consuming the unfermented rooibos $(93.4 \mathrm{mg} /$ day $)$ and fermented rooibos $(73.1 \mathrm{mg} /$ day). Intake of both the derived supplement and unfermented rooibos restored the $t$-BHP-induced reduction and increased $(P<0.05)$ the antioxidant capacity status of the liver, while not impacting on lipid peroxidation. The rooibos herbal tea did not affect the hepatic antioxidant enzymes, except fermented rooibos that caused a decrease $(P<0.05)$ in superoxide dismutase activity. This study confirms rooibos herbal tea as good dietary antioxidant sources and, in conjunction with its many other components, offers a significantly enhanced antioxidant status of the liver in an induced oxidative stress situation.
\end{abstract}

\section{Introduction}

There has been a steady increase in the popularity of polyphenols/flavonoids and other dietary products as health and wellness promoting agents against excess of free radicals. Epidemiological studies have demonstrated an inverse correlation between the consumption of diets rich in flavonoids and decreased cellular damage via direct radical scavenging of reactive species, chelating of metal ions, or challenging enzymatic systems responsible for free radical production [1-3]. Some of these compounds can activate phase II detoxification enzymes and modify physiological and biotransformation reactions, thus enhancing the xenobiotic detoxification process [4]. Much of the information supporting the usefulness of these nutraceuticals to reverse oxidative stress damage was mainly anecdotal, but in recent years, systematic in vitro, animal and human studies have greatly enhanced our knowledge regarding the bioavailability, safety, and efficacy of these compounds as well as their underlying mechanisms of action against oxidative stress [5-8].

Hepatic tissue is greatly exposed to oxidative stress due to its important role in the regulation of various physiological processes including metabolism of carbohydrates and fats, synthesis of bile acids and coagulation proteins, and detoxification of endogenous and xenobiotic compounds among others, exposing itself to free radical injury as a result of sustained exposure to numerous potential toxicants. This damage by free radicals may result from direct or indirect attacks on essential biomolecules leading to the development of diseases such as cancer, nonalcoholic fatty liver disease, and many others [9-12].

Aspalathus linearis, commonly known as rooibos, is a shrub native to the Cederberg region in the Western Cape Province of South Africa [13,14]. It is frequently used to make a mild tasting tisane rich in polyphenol antioxidants but with no caffeine and very little tannins; it is also claimed 
to cure insomnia, allergies, and nervous breakdown as well as improve the appetite $[15,16]$. Currently, many of these claims are at various stages of formal substantiation; however, recent scientific endeavors suggest that rooibos may confer various antioxidant-associated health benefits including antimutagenic, anticarcinogenic, anti-inflammatory, and antiviral properties and antiatherosclerotic effects [16-19]. Some of these health benefits were recently confirmed in humans where the consumption of fermented rooibos improved the lipid profile as well as redox status in adults at risk of developing cardiovascular diseases [20].

Previous and recent studies have demonstrated Aspalathus linearis as a good source of unique phenolic compounds exhibiting its protective effect in various tissues $[10,21,22]$. To date, no comparative study has been done that includes a rooibos-derived commercial supplement and compares it to the two forms of rooibos herbal tea, fermented and unfermented, to determine their oxidative stress modulatory effect in liver tissue. The rooibos-derived commercial supplement used in the current study contains $175 \mathrm{mg}$ of a $20 \%$ aspalathin-rich extract, $500 \mu \mathrm{g}$ vitamin $\mathrm{A}$, $150 \mathrm{mg}$ vitamin C, $5 \mathrm{mg}$ Vitamin $\mathrm{E}$, and $25 \mu \mathrm{g}$ selenium that are hoped to confer a synergistic interaction amongst them. Aspalathin is the main polyphenol present in Aspalathus linearis and recent studies have shown that the rooibos antioxidative properties are, in part, attributed to the strong ability to quench radicals generated in the water phase, which can also confer high thermal stability against deep frying $[17,18]$. Vitamin $C$ is considered a first line of antioxidant defence system and as the major water soluble antioxidant, acting synergistically with $\alpha$-tocopherol by reducing $\alpha$ tocopheroxyl radicals rapidly to regenerate $\alpha$-tocopherol, and possibly inhibits $\alpha$-tocopheroxyl-mediated radical propagation [23, 24]. Vitamin $\mathrm{E}$ is the most bioactive of the isomers and has received much attention due to its radical-scavenging capability in the lipophilic phase [24]. This mixture of molecules acting at different phases and levels of the biological defence system may be more efficient than an isolated compound. These claims were supported in some studies. For example, Bakker et al. [25] studied the anti-inflammatory effects of a dietary mix of resveratrol, green tea extract, tomato extract, $\alpha$-tocopherol, vitamin $\mathrm{C}$, and omega-3 polyunsaturated fatty acids in overweight men where plasma levels of adiponectin, anti-inflammatory and antidiabetic hormone secreted by adipocytes, were increased by $7 \%$, while C-reactive protein was unaffected, oxidative stress biomarkers (isoprostanes and uric acid) were decreased, and concurrently endothelial function was improved.

In the current study tert-butyl hydroperoxide ( $t$-BHP), a short-chain organic hydroperoxide commonly used in models for oxidative injury in various tissues including hepatic tissue, was used as a free radical generator. After metabolic activation, metabolites such as $t$-butoxyl and $t$ methyl radicals may cause permeabilisation of cell membranes, DNA damage, and depletion of glutathione via a variety of mechanisms [26-28].
This study aimed to assess the redox modulation of fermented and unfermented rooibos herbal tea and a rooibosderived commercial supplement in the liver tissue of $t$-BHPinduced oxidative stress male Wistar rats.

\section{Material and Methods}

2.1. Chemicals. All chemicals used were of analytical grade purity. The Folin Ciocalteu reagent (FCR), 4dimethylaminocinnamaldehyde (DMACA), hydrochloric acid $(\mathrm{HCl})$, methanol $(\mathrm{MeOH})$, ethanol (EtOH), phosphoric acid $\left(\mathrm{H}_{3} \mathrm{PO}_{4}\right)$, sodium acetate, glacial acetic acid, ferric chloride, disodium hydrogen orthophosphate dehydrate and dihydrogen orthophosphate-1-hydrate, 2-2'-azino-bis(3-ethylbenzothiazoline-6-sulfonic acid $\left(\mathrm{ABTS}^{\bullet+}\right)$, potassium peroxodisulphate, trichloroacetic acid (TCA), 1,1,3,3,-tetraethoxypropane (TEP), chloroform, cyclohexane, malondialdehyde bisdiethyl acetal (MDA), perchloric acid (PCA), thiobarbituric acid, and sulfuric acid were obtained from Merck (SA), while gallic acid, quercetin, ascorbic acid, fluorescein (FI) sodium salt, 2,2'-azobis(2-methylpropionamidine)dihydrochloride

(AAPH), Na-Pi, 0.5\%, Triton X-100, 30\% hydrogen peroxide, potassium dihydrogen orthophosphate $\left(\mathrm{KH}_{2} \mathrm{PO}_{4}\right)$, sodium phosphate, ethylenediaminetetraacetic acid (EDTA), glutathione reductase, $\beta$-NADPH, sodium azide, $\mathrm{NaH}_{2} \mathrm{PO}_{2}$, 6-hydroxydopamine, $1.6 \mathrm{mM}$ (6-HD), diethylenetriaminepentaacetic acid (DETAPAC), 5,5' dithiobis (2-nitrobenzoic acid) (DTNB), 1-methyl-2vinylpyridinium (M2VP) glutathione reductase (GR), reduced glutathione $(\mathrm{GSH})$, iron chloride hexahydrate $\left(\mathrm{FeCl}_{3} 6 \mathrm{H}_{2} \mathrm{O}\right)$, 2,4,6-tri[2-pyridyl]-s-triazine (TPTZ), and 6-hydroxy-2,5,7,8-tetramethylchroman-2-carboxylic acid (trolox) were obtained from Sigma-Aldrich (SA).

2.2. Preparation of Rooibos Beverages and Supplement. Aqueous extracts of the various plant materials, namely, fermented and unfermented rooibos, were freshly prepared by adding the plant material (superior grade) to freshly boiled tap water at a concentration of $2 \mathrm{~g} / 100 \mathrm{~mL}$ (concentrations identical to those used in previous studies) [22, 29]. Firstly, the herbal tea was brewed for $30 \mathrm{~min}$ at room temperature before filtering (Whatman N 4), cooled, and then served to the experimental animals in their water bottles. Aliquots of the freshly prepared rooibos herbals tea and rooibos-derived commercial supplement were also stored at $-20^{\circ} \mathrm{C}$ for quantitative antioxidant analyses. The rooibos herbal beverages were freshly prepared every second day. Fermented and unfermented rooibos plant material (superior grade) was a generous gift from Rooibos Ltd. (Clanwilliam, South Africa). The rooibos-derived commercial supplement was crushed using a pestle and mortar and prepared at a concentration of $2 \mathrm{~g} / 100 \mathrm{~mL}$. The rooibos-derived commercial supplement was purchased from a local drug store (Cape Town, South Africa).

2.3. Experimental Animals and Diet. Following ethical approval (CPUT/HWS-REC 2010/A001) granted by the Faculty of Health and Wellness Sciences Research Ethics 
Committee, Cape Peninsula University of Technology, forty male Wistar rats obtained from the Animal Unit of the University of Cape Town (South Africa) weighing 120-150 g were randomly allocated to one of the four study groups. Each group, consisting of ten rats each, was maintained using standard care as described by Awoniyi et al. [22]. Briefly, animals were housed in Perspex houses with stainless wire-bottomed cages in a controlled environment of 24$25^{\circ} \mathrm{C}$ with 12 hours light-dark cycles and $50 \%$ humidity for 1 week. The rats had free access to standard rat chow, the various beverages (fermented and unfermented rooibos and the rooibos-derived commercial supplement), and tap water for the control group for 10 weeks as sole source of drinking fluid. No negative control was included in the current study to ascribe model functionability, as the $t$-BHP concentration used in this study was similar to that used in other studies where it was able to generate oxidative stress $[28,30,31]$. Body weight was monitored twice a week till the end of the experiment. Oxidative stress was induced in all animals via an intraperitoneal (i.p.) injection of $30 \mu$ mole of $t$-BHP per $100 \mathrm{~g}$ body weight in the final two weeks of the study. At the end of the intervention, the animals were sacrificed under pentobarbital anaesthesia (i.p. $0.4 \mathrm{~mL} / \mathrm{kg}$ body weight). Thereafter, livers were excised, immediately snap-frozen in nitrogen, and then stored at $-80^{\circ} \mathrm{C}$. Prior to analyses, the livers were weighed and homogenised according to the specific assay requirements.

2.4. Assessment of Phenolic Contents. Assessment of the phenolic contents of the rooibos herbal tea and rooibosderived commercial supplement included measurements of total polyphenols (TPs), flavanols, and flavonols. The total polyphenol content was determined according to the method described by Waterhouse [32]. Briefly, an $800 \mathrm{mg} / \mathrm{L}$ gallic acid stock standard solution was used to prepare the standard series of $0,20,50,100,250$, and $500 \mu \mathrm{g} / \mathrm{L}$ using distilled water as the diluent. The Folin working solution was prepared by diluting $1 \mathrm{~mL}$ of Folin Ciocalteu reagent (FCR) with $9 \mathrm{~mL}$ of distilled water. The reaction mixture in each 96-microplate well consisted of $25 \mu \mathrm{L}$ of standard or sample, $125 \mu \mathrm{L}$ FCR, and $100 \mu \mathrm{L}$ of sodium carbonate $(7.5 \%)$ added after $5 \mathrm{~min}$ and read in a Multiskan Spectrum plate reader (Thermo Electron Corporation, USA) set at $25^{\circ} \mathrm{C}$ and $765 \mathrm{~nm}$. The polyphenol content was expressed as $\mathrm{mg}$ gallic acid equivalents per litre of beverage or supplement (mg GAE/L). The flavanol and flavonol contents of the fermented and unfermented rooibos herbal tea and rooibos-derived commercial supplement were determined colorimetrically at $640 \mathrm{~nm}$ and $360 \mathrm{~nm}$, using the methods described by Treutter et al. [33] and Mazza et al. [34], respectively, with flavanol contents expressed as $\mathrm{mg}$ catechin equivalents (CE) per litre and flavonol contents expressed as mg quercetin equivalents $(\mathrm{QE})$ per litre.

2.5. Estimation of Antioxidant Capacity. The antioxidant capacity of the various rooibos herbal tea and rooibosderived commercial supplement consumed in the study was determined using the oxygen radical absorbance capacity (ORAC) assay, while that of the rat liver tissue was assessed using three different assays, that is, the ferric reducing antioxidant power (FRAP) assay, the ORAC assay, and the ABTS+ assay expressed as trolox equivalent antioxidant capacity (TEAC). The FRAP assay was used as described by Benzie and Strain [35] to assess the ability of the sample to reduce the 2,4,6-tri[2-pyridyl]-s-triazine $\left(\mathrm{Fe}^{3+}\right)$ to the ferrous $\left(\mathrm{Fe}^{2+}-\mathrm{TPTZ}\right.$ complex) state at low $\mathrm{pH}$, forming an intense blue-coloured complex with optimal absorbance at $593 \mathrm{~nm}$, while the ABTS+ assay was used as described by Re and coworkers [36]. For the FRAP and ABTS+ assays, $300 \mathrm{mg}$ subsamples of the liver tissue were homogenized in $1.2 \mathrm{~mL}$ of freshly prepared $75 \mathrm{mM}$ phosphate buffer $(\mathrm{pH}$ 7.0) following centrifugation (Eppendorf 5810R, Eppendorf, Germany) for $30 \mathrm{~min}$ at $4000 \mathrm{rpm}, 4^{\circ} \mathrm{C}$, and used as is. Data analyses were carried out using Microsoft Excel 2003 based on a calibration curve plotted using a standard dilution series of ascorbic acid (AA) and trolox (T), respectively. The ORAC assay, as described by Ou et al. [37], was used to estimate the antioxidant capacity of the various rooibos herbal tea types (required a 20 -fold dilution in phosphate buffer) and liver tissues (required proteins to be precipitated with 5\% PCA and also a 20 -fold dilution in phosphate buffer); each reaction mixture consisted of $12 \mu \mathrm{L}$ of proteinfree sample or standard and $138 \mu \mathrm{L}$ of fluorescein (FI) and was initiated by the addition of $50 \mu \mathrm{L}$ of $2,2^{\prime}$-azobis(2methylpropionamidine)dihydrochloride (AAPH) solution. The activity was determined using the regression equation $y=a x^{2}+b x+c$. All assays were done in triplicate and results were expressed as $\mu$ mole AAE/g tissue, $\mu$ mole TE/g for the tissue samples, and $\mu$ mole $\mathrm{TE} / \mathrm{L}$ for the rooibos herbal tea and derived supplement.

2.6. Estimation of Lipid Peroxidation Products. Estimation of lipid peroxidation products was done by measuring thiobarbituric acid reactive substances (TBARS), which reflect the production of malondialdehyde (MDA), a product of lipid oxidation. The use of high-performance liquid chromatography (HPLC) with fluorescence detection circumvents some of the limitations of previous methods and was therefore used in the current study. The method described by Recknagel and Glende [38] was used, with the MDA-TBA adduct analysed using HPLC. Briefly, the thawed aliquots $(250 \mu \mathrm{L})$ of liver tissue homogenates were pipetted into $1.5 \mathrm{~mL}$ reaction tubes containing $50 \mu \mathrm{L}, 6 \mathrm{M}$ sodium hydroxide and incubated at $60^{\circ} \mathrm{C}$ for $30 \mathrm{~min}$ for protein hydrolysis. Thereafter, $125 \mu \mathrm{L}$ of $35 \%$ perchloric acid was added to precipitate proteins, after the mixture was centrifuged at $4^{\circ} \mathrm{C}, 2800 \mathrm{rpm}$ for $10 \mathrm{~min}$. Supernatants $(100 \mu \mathrm{L})$ were pipetted into new reaction tubes containing $250 \mu \mathrm{L} 40 \mathrm{mM}$ TBA and $750 \mu \mathrm{L} 0.2 \mathrm{M}$ orthophosphoric acid. This mixture was boiled at $100^{\circ} \mathrm{C}$ for $1 \mathrm{hr}$, cooled on ice, and centrifuged (Eppendorf AG 5810R, Germany) at $14000 \mathrm{rpm}$ for $4 \mathrm{~min}$. The MDA standard was prepared by dissolving $25 \mu \mathrm{L}$ 1,1,3,3-tetraethoxypropane (TEP) in $100 \mathrm{~mL}$ water to provide a $1 \mathrm{mM}$ stock solution. Working standards were prepared by mixing $1 \mathrm{~mL}$ TEP stock solution in $50 \mathrm{~mL}$ $1 \%$ sulphuric acid incubated for $2 \mathrm{hr}$ at room temperature. The resulting MDA standard $(20 \mu \mathrm{mole} / \mathrm{L})$ was further diluted with $1 \%$ sulphuric acid to final concentrations of 
$0.5,1,2,5$, and $10 \mu \mathrm{M}$ for determination of the standard curve for the estimation of MDA. The reaction mixture $(20 \mu \mathrm{L})$ was injected into an Agilent Technology 1200 Series (Germany) HPLC system for separation of the MDA-TBA complex. The mobile phases used were as follows: A: $100 \%$ methanol and B: potassium dihydrogen phosphate $\left(\mathrm{KH}_{2} \mathrm{PO}_{4}\right)$ buffer $(50 \mathrm{mM}$, $5.8 \mathrm{pH})$ in a ratio $(\mathrm{A}: \mathrm{B}) 60 \%: 40 \%$. A flow rate of $0.8 \mathrm{~mL} / \mathrm{min}$ and fluorescent detection with excitation wavelength set at $532 \mathrm{~nm}$ and emission wavelength set at $552 \mathrm{~nm}$ were used. Results were expressed as nmole/mg in the tissue extract sample.

2.7. Measurement of Antioxidant Enzymes. The three enzymes, namely, catalase (CAT), glutathione peroxidase $(\mathrm{GPx})$, and superoxide dismutase (SOD), were measured to determine liver antioxidant enzymes activity. The tissue samples were prepared by homogenising $300 \mathrm{mg}$ in $2 \mathrm{~mL}$ of phosphate buffer followed by centrifugation at $15000 \mathrm{~g}$, $10 \mathrm{~min}, 4^{\circ} \mathrm{C}$, and subsequent separation of the supernatant. CAT activity was assessed spectrophotometrically by the modified method of Ellerby and Bredesen [39]. GPx activity was determined using the method of Flohé and Günzler [40] and SOD as described by Ellerby and Bredesen [39]. The two assays were done in duplicate and enzyme activity of catalase in the sample was calculated based on the rate of decomposition of hydrogen peroxide which is proportional to the reduction of absorbance at $240 \mathrm{~nm}$ wavelength, while glutathione peroxidase activity in the sample was calculated based on the rate of decrease in absorbance at $340 \mathrm{~nm}$ wavelength over the time using the molar extinction coefficient of glutathione peroxidase and corrected for path length. The estimation of SOD activities was done in duplicate using a standard curve with SOD at different concentrations. All results were expressed as $\mu \mathrm{mole} / \mathrm{min} / \mu \mathrm{g}$ protein.

2.8. Protein Determination. Protein concentrations of all liver homogenates and/or supernatants were determined using the Pierce bicinchoninic acid (BCA) protein assay, with bovine serum albumin as standard. Results were expressed as mg protein/mL tissue supernatant of homogenate.

2.9. Statistical Analyses. Results were expressed as a means \pm standard deviation (S.D) for each group tested. Two- way analysis of variance (ANOVA) complemented with Duncan Bonferroni Alpha for multiple comparison and Spearman's Rank Correlation Coefficient tests were used to compare the groups and assess the strength of their associations. In all analyses, the level of significance was set at $P<0.05$.

\section{Results}

3.1. Antioxidant Profile of the Rooibos Beverages and Supplement. Results of the polyphenol, flavonol, and flavanol contents and oxygen radical absorbance capacity of the rooibos herbal tea and rooibos-derived commercial supplement (DCRS) are shown in Table 1. The DCRS contained significantly $(P<0.05)$ higher levels of total polyphenols, which may reflect the purified and rich phenolic compound present in the mixture, when compared to the fermented and unfermented rooibos herbal tea, while as expected the DCRS also presented the highest antioxidant capacity, as determined by the ORAC assay.

3.2. Daily Beverage Intake and Body Weight. Neither the rooibos herbal tea nor rooibos-derived commercial supplement was associated with any changes in body weight gains, Table 2. Rats consuming the derived supplement showed a significantly $(P<0.05)$ lower daily fluid intake, but this did not impair significantly their body weight gains. The highest $(P<0.05)$ daily polyphenol intake was achieved by rats consuming the derived supplement, followed by rats consuming the unfermented rooibos and then the fermented rooibos. When considering their daily ORAC intake, it was the rats consuming the unfermented rooibos that showed the highest $(P<0.05)$ intake, while the ORAC intake was similar $(P>0.05)$ for the fermented rooibos and derived supplement groups. Although the daily fluid intake of rats consuming the fermented and unfermented rooibos was higher $(P<0.05)$, their daily intake of polyphenols was lower $(P<0.05)$ when compared to the daily intake of the derived supplement. It is known that the absorption phase impacts on biodisponibility of individual polyphenol content [41, 42]. Rats consuming the rooibos herbal tea had the highest $(P<0.05)$ flavonol intake when compared to the group consuming the derived supplement.

3.3. Hepatic Antioxidant Capacity. The comparative data of the hepatic antioxidant capacity (AOC) in the liver of rats consuming the rooibos herbal tea and rooibos-derived commercial supplement after $t$-BHP-induced oxidative stress are shown in Table 3 . With the exception of FRAP activity in all the groups, there was a significant $(P<0.05)$ increase in the hepatic AOC in the rats consuming the unfermented rooibos as well as the derived supplement when compared to the water control and fermented rooibos groups. These increases were significant $(P<0.05)$ when considering ORAC (unfermented rooibos and the derived supplement groups) and ABTS+ (derived supplement group).

3.4. Lipid Peroxidation. The effect of the dietary antioxidant interventions on $t$-BHP-induced oxidative damage using MDA as marker of lipid peroxidation is shown in Table 4. None of the rooibos interventions had any significant effect on the $t$-BHP-induced MDA elevation in the liver when compared to the control group consuming water. However, a decreasing trend in this marker was noted in all the groups, with the group consuming the rooibos-derived commercial supplement showing the lowest value $(121 \mathrm{nmole} / \mathrm{mg} \pm 22$ versus $153 \mathrm{nmole} / \mathrm{mg} \pm 34$ for the control).

3.5. Hepatic Antioxidant Enzymes. Data on the activities of hepatic antioxidant enzymes of rats consuming the various rooibos herbal tea and rooibos-derived commercial supplement are presented in Table 5. It has been reported that treatment with $t$-BHP significantly reduces glutathione-related 
TABle 1: Polyphenol content and antioxidant activity of the rooibos herbal tea and rooibos-derived commercial supplement.

\begin{tabular}{|c|c|c|c|}
\hline & \multicolumn{3}{|c|}{ Aqueous formulations } \\
\hline & Fermented rooibos & Unfermented rooibos & Rooibos-derived commercial supplement \\
\hline Total polyphenols (mg GAE/L) & $981 \pm 118^{\mathrm{a}}$ & $1354 \pm 62^{\mathrm{b}}$ & $4836 \pm 244^{\mathrm{c}}$ \\
\hline Flavanols (mg CE/L) & $39 \pm 8^{\mathrm{a}}$ & $92 \pm 3^{\mathrm{b}}$ & $18 \pm 10^{\mathrm{a}}$ \\
\hline Flavonols (mg QE/L) & $299 \pm 49^{\mathrm{a}}$ & $247 \pm 19^{\mathrm{b}}$ & $181 \pm 22^{\mathrm{c}}$ \\
\hline ORAC $(\mu$ mole TE/L $)$ & $14557 \pm 905^{\mathrm{a}}$ & $20889 \pm 1281^{b}$ & $33768 \pm 2322^{c}$ \\
\hline
\end{tabular}

Values are expressed as mean of three measurements for each aqueous sample \pm STD. TE: trolox equivalents; AAE: ascorbic acid equivalents; CE: catechin equivalents; QE: quercetin equivalents; GAE: gallic acid equivalents. Similar letters in rows denote lack of significant difference $(P>0.05)$, but if letters differ then $P<0.05$.

TABLE 2: Body weight gain and daily fluid intake of tert-butyl hydroperoxide-induced oxidative stress rats.

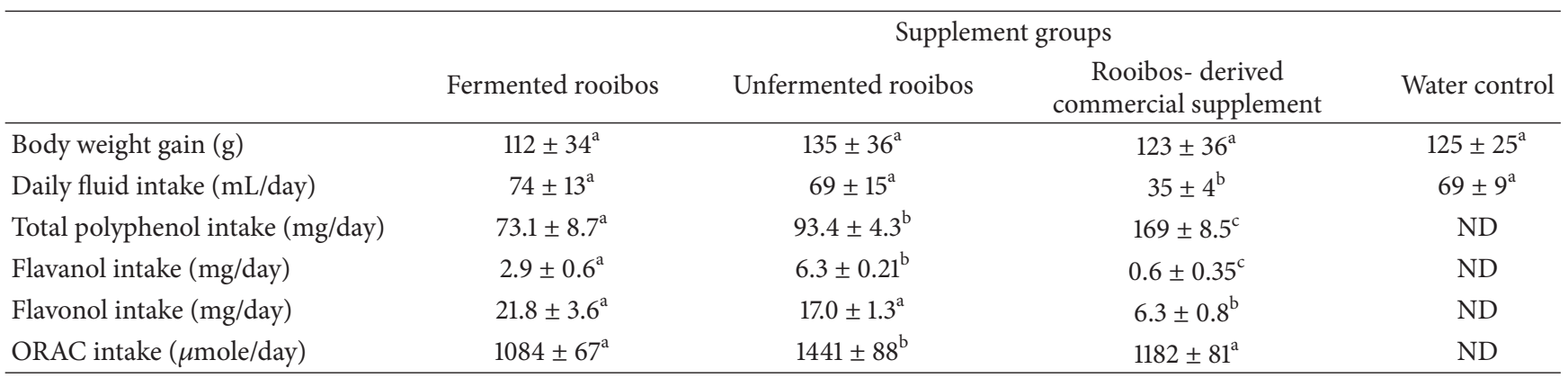

Values are expressed as mean \pm STD of 10 rats per group. Similar letters in rows denote lack of significant difference $(P>0.05)$, but if letters differ then $P<0.05$. The daily intake is based on the respective beverage profiles provided in Table 1. ND: not done as these rats consumed tap water. Above intakes were based on fluid intake alone.

TABLE 3: Hepatic antioxidant capacity of rats consuming the rooibos herbal tea and rooibos-derived commercial supplement after tert-butyl hydroperoxide-induced oxidative stress.

\begin{tabular}{|c|c|c|c|c|}
\hline \multirow[b]{2}{*}{ Antioxidant activity } & \multicolumn{4}{|c|}{ Supplement groups } \\
\hline & Fermented rooibos & Unfermented rooibos & $\begin{array}{l}\text { Rooibos-derived } \\
\text { commercial supplement }\end{array}$ & Water control \\
\hline FRAP $(\mu$ mole AAE/g) & $5.36 \pm 0.5^{\mathrm{a}}$ & $4.60 \pm 0.5^{\mathrm{a}}$ & $5.49 \pm 2.2^{\mathrm{a}}$ & $5.01 \pm 0.7^{\mathrm{a}}$ \\
\hline ORAC ( $\mu$ mole TE/g) & $4.55 \pm 1.4^{\mathrm{a}}$ & $6.53 \pm 2.1^{b}$ & $6.38 \pm 1.2^{\mathrm{b}}$ & $4.00 \pm 2.1^{\mathrm{a}}$ \\
\hline TEAC ( $\mu$ mole TE/g) & $41.3 \pm 2.0^{\mathrm{a}}$ & $38.9 \pm 2.4^{\mathrm{a}}$ & $41.9 \pm 1.3^{\mathrm{b}}$ & $37.4 \pm 5.4^{\mathrm{a}}$ \\
\hline
\end{tabular}

Values are expressed as mean \pm STD of 10 rats per group. Similar letters in rows denote lack of significant difference $(P>0.05)$, but if letters differ then $P<$ 0.05. TE: trolox equivalents; AAE: ascorbic acid equivalents.

TABLE 4: Effect of rooibos herbal tea and rooibos-derived commercial supplement on lipid peroxidation in the liver of tert-butyl hydroperoxide-induced oxidative stress rats.

\begin{tabular}{lccc}
\hline Lipid peroxidation levels & Fermented rooibos & Unfermented rooibos & $\begin{array}{c}\text { Supplement groups } \\
\text { Rooibos-derived } \\
\text { commercial supplement }\end{array}$ \\
\hline TBARS (nmole/mg) & $135 \pm 27^{\mathrm{a}}$ & $144 \pm 21^{\mathrm{a}}$ & $121 \pm 22^{\mathrm{a}}$ \\
\hline
\end{tabular}

Values are expressed as mean \pm STD of 10 rats per group. Similar letters in rows denote lack of significant difference $(P>0.05)$, but if letters differ then $P<$ 0.05 .

TABLE 5: Hepatic antioxidant enzyme activity in $t$-BHP-induced oxidative stress rats consuming the rooibos herbal tea and rooibos-derived commercial supplement.

\begin{tabular}{|c|c|c|c|c|}
\hline \multirow{2}{*}{$\begin{array}{l}\text { Antioxidant activity } \\
(\mu \mathrm{mole} / \mathrm{min} / \mu \mathrm{g} \text { protein })\end{array}$} & \multicolumn{4}{|c|}{ Supplement groups } \\
\hline & Fermented rooibos & Unfermented rooibos & $\begin{array}{c}\text { Rooibos-derived } \\
\text { commercial supplement }\end{array}$ & Water control \\
\hline Catalase & $0.153 \pm 0.06^{\mathrm{a}}$ & $0.179 \pm 0.04^{\mathrm{a}}$ & $0.190 \pm 0.09^{\mathrm{a}}$ & $0.261 \pm 0.13^{\mathrm{a}}$ \\
\hline Glutathione peroxidase & $0.240 \pm 0.06^{\mathrm{a}}$ & $0.233 \pm 0.06^{\mathrm{a}}$ & $0.267 \pm 0.05^{\mathrm{a}}$ & $0.310 \pm 0.19^{\mathrm{a}}$ \\
\hline Superoxide dismutase & $0.019 \pm 0.01^{\mathrm{b}}$ & $0.028 \pm 0.01^{\mathrm{a}}$ & $0.024 \pm 0.02^{\mathrm{a}}$ & $0.041 \pm 0.02^{\mathrm{a}}$ \\
\hline
\end{tabular}

Values are expressed as mean \pm STD of 10 rats per group. Similar letters in rows denote lack of significant difference $(P>0.05)$, but if letters differ then $P<$ 0.05 . 
enzyme activities [43]. In the current study, when compared to the control group, none of the dietary antioxidant interventions showed any significant changes in the hepatic CAT and GPx activities. When considering the hepatic SOD activity, the group consuming the fermented rooibos showed a significant $(P<0.05)$ decrease in the hepatic activity of this enzyme when compared to the control group consuming water. In the same intervention group, correlation coefficient found that superoxide dismutase was positively correlated with ORAC and TEAC $\left(r^{2}=.661, P=0.038\right.$ and $r^{2}=.867$, $P=0.001$, resp.).

\section{Discussion}

There is substantial evidence from human and experimental animal studies that biomarkers of oxidative damage present in plasma, urine, and cells are increased in subjects with risk factors for or displaying certain disease conditions, as well as in certain physiological conditions, such as in intense and prolonged exercise. For example, the in vivo markers for lipid peroxidation, $\mathrm{F}_{2}$-isoprostanes, and their metabolites are increased in liver and neurological disorders and in individuals with CVD risk factors, such as cigarette smoking, diabetes mellitus, obesity, and hypercholesterolemia [44-46]. Disease developing from oxidative injury to vital biological macromolecules is thus a possibility and the dietary intake of, or supplementation with, antioxidants as a means to reduce the risk for disease development is feasible. However, it is important to note that polyphenols are a heterogenous group of compounds, and their antioxidant properties may be affected by their absorption, distribution, retention, metabolism, and related enzymatic levels leading to the production of putative metabolites with different antioxidant activities $[23,42]$.

The current study confirms that fermented rooibos herbal tea has a lower total polyphenol content when compared to its unfermented counterpart, as the fermentation process (an oxidative environment) is known to decrease the total polyphenol concentration due to structural and enzymatic changes taking place $[16,47]$. The rooibos-derived commercial supplement (RDCS) showed a significantly higher polyphenol content due to the presence of the aspalathin-rich extract. During fermentation, the dihydrochalcone content of rooibos is decreased with less than $7 \%$ of the original aspalathin content remaining in fermented rooibos [47]. The presence of this extract as well as the various antioxidant vitamins and trace elements in the rooibos-derived supplement explains its higher antioxidant capacity when compared to the rooibos herbal tea. Antioxidants are known to interact with each other sometimes synergistically through overlapping or complementary effects but this may not be the case in a dynamic and complex biological system where compounds competing for the same functional site may result in inhibition [48-50]. Future studies should be aimed at unravelling these complex interactions to further elucidate the current findings.

The hepatic antioxidant status of the $t$-BHP-induced oxidative stress rats was evaluated using three different antioxidant capacity assays, that is, ORAC, FRAP, and ABTS+, as these assays use different endpoint measurements and quantification approaches $[51,52]$. Also, it is important to keep in mind that biological systems are complex and characterised by multiple mechanisms involved in oxidative stress. On the basis of that, previous authors have recommended a battery of assays rather than a single assay approach, keeping in mind the specific research question to be addressed $[23,53-55]$. In the current study, none of the intervention groups could significantly influence the hepatic FRAP measurement. However, the intake of unfermented rooibos and the rooibos-derived commercial supplement significantly restored the $t$-BHP-induced reduction of AOC and increased the hepatic antioxidant status with regard to ORAC and TEAC levels. A previous study by Marnewick et al. [18] also showed that unfermented rooibos restored and enhanced the injured livers' ORAC levels in rats exposed to diethylnitrosamine (DEN). In 2010 Villaño et al. [56] showed for the first time that both fermented and unfermented rooibos teas are able to increase plasma antioxidant defences in a human study. ORAC measures the degree of inhibition of peroxyl-radical-induced oxidation by a compound and rooibos's scavenging capacity or antioxidant activity may account for potential effects of secondary products. From the results obtained in this study, it would be difficult to deduce exactly which group of compounds was responsible for this enhanced antioxidant capacity, but it could be speculated that it is due to the polyphenolic compounds, most likely those present at the levels found in the unfermented rooibos. When ingested, dietary flavonoids may cause different benefits as those displayed in vitro as a result of absorption, extensive phase III metabolism, and rapid excretion. Results from a previous study confirmed the presence of $O$-linked methyl, sulphate, and glucuronide metabolites of aspalathin in urine of humans that consumed rooibos [57]. These putative metabolites may display different biological activities and could explain, in part, the differences shown in the current study. Unfortunately information regarding the metabolic changes to phenolic compounds present in rooibos herbal tea is scarce and further studies are needed to elucidate this protective effect and the possible role single and/or combinations of compounds pay in the health promoting properties of plant phytochemicals.

The hepatic lipid peroxidation status was assessed using HPLC measuring MDA which has been widely used for determining oxidative stress in mammalian tissues. In this study neither the rooibos herbal tea nor rooibos-derived commercial supplement impacted significantly on the lipid peroxidation status, although a declining trend of TBARS levels in the liver tissues of all intervention groups was noticed. It could be argued that this assay lacks specificity and that the estimates are unlikely to effectively reflect the oxidative injury [58]; however, it is important to note that reports on the effect of polyphenolic intake on lipid peroxidation have demonstrated controversial outcomes. Several studies have suggested a decreased lipid peroxidation level in plasma and hepatic tissue, while others have not [59-61]. Hodgson et al. [62] reported that green tea did not decrease the level of oxidative lipid damage, as measured by urinary 
$\mathrm{F}_{2}$-isoprostanes excretion. Also, the unconjugated flavanol levels in plasma are in the range of $1 \mu \mathrm{M}$, which may not be sufficient to yield a greater antioxidant capacity in a complex and dynamic biological system [63]. Future studies should be aimed at elucidating the dose-response relationship of rooibos flavonoids.

As antioxidant enzymes are part of the endogenous defence system and may protect against oxidative stress, none of the rooibos intervention groups showed any further significant increase in the hepatic CAT and GPx activities, when compared to the control group. This supports the findings of Marnewick and coworkers [18] who could not show any increased enzyme activities in liver tissue from rats treated with DEN and consuming the rooibos herbal tea. However, in the present study the hepatic SOD activity in the group consuming the fermented rooibos showed a significant $(P<0.05)$ decrease when compared to the control group consuming water. An increased enzyme activity may be prompted by increased oxidative stress in a negative feedback-like relation, while, conversely, a decreased enzyme activity may reflect a favourable oxidative status balance; that is, enzyme levels decrease as the rate of oxyradicals formation (requiring neutralization) diminishes [64]. In the current study, the positive correlation observed between the hepatic SOD activity and antioxidant capacity (ORAC and TEAC) may strengthen claims that different antioxidants act at different sites as a network to ascribe system efficacy. However, the extent of these correlations among other organs is still to be determined. Similar links between enzymes and antioxidant capacity measures were observed by Dudonné et al. [65] in plants extracts, but in animals models and human studies, correlations between the methods discussed to assess the effect of rooibos herbal tea are not well reported in scientific literature, which suggest the need for further studies that could enhance our current understanding on these matters as a tool for effective study design and interventions.

\section{Conclusion}

We reported a higher concentration of total polyphenol content in the derived rooibos supplement that may have accounted for the significant higher hepatic oxygen radical antioxidant capacity and the declined trend of lipid peroxidation status in the $t$-BHP-treated rats. Rats consuming the unfermented rooibos herbal tea also showed a significantly enhanced hepatic antioxidant status, while those consuming the fermented rooibos did not. A significant correlation between SOD, ORAC, and TEAC was also found in the livers of rats consuming the fermented rooibos herbal tea. The use of several methodologies to assess the oxidative status displayed results which would otherwise have been missed if only single assays were used. Results from this study suggest that the daily intake of unfermented rooibos herbal tea or a derived commercial rooibos supplement may benefit human health by providing the liver with an enhanced antioxidant capacity to reduce damage induced by toxicants.

\section{Conflict of Interests}

The authors declare that there is no conflict of interests regarding the publication of this paper.

\section{Acknowledgments}

The authors thank the CPUT Research Directorate for the research and financial support and Mr. D. Awoniyi, Mr. M. Macharia, and colleagues in the Oxidative Stress Research Centre for many useful discussions.

\section{References}

[1] R. J. Nijveldt, E. van Nood, D. E. C. van Hoorn, P. G. Boelens, K. van Norren, and P. A. M. van Leeuwen, "Flavonoids: a review of probable mechanisms of action and potential applications," The American Journal of Clinical Nutrition, vol. 74, no. 4, pp. 418425, 2001.

[2] O. Firuzi, L. Fuksa, C. Spadaro et al., "Oxidative stress parameters in different systemic rheumatic diseases," Journal of Pharmacy and Pharmacology, vol. 58, no. 7, pp. 951-957, 2006.

[3] A. Lukačínová, J. Mojžiš, R. Beňačka, O. Rácz, and F. Ništiar, "Structure-activity relationships of preventive effects of flavonoids in alloxan-induced diabetes mellitus in rats," Journal of Animal and Feed Sciences, vol. 17, no. 3, pp. 411-421, 2008.

[4] P. Saha and S. Das, "Regulation of hazardous exposure by protective exposure: modulation of phase II detoxification and lipid peroxidation by Camellia sinensis and Swertia chirata," Teratogenesis Carcinogenesis and Mutagenesis, vol. 23, supplement 1, pp. 313-322, 2003.

[5] L. A. Pham-Huy, H. He, and C. Pham-Huy, "Free radicals, antioxidants in disease and health," International Journal of Biomedical Science, vol. 4, no. 2, pp. 89-96, 2008.

[6] E. G. de Mejia, M. V. Ramirez-Mares, and S. Puangpraphant, "Bioactive components of tea: cancer, inflammation and behavior," Brain, Behavior, and Immunity, vol. 23, no. 6, pp. 721-731, 2009.

[7] D. E. Stevenson and R. D. Hurst, "Polyphenolic phytochemicals-just antioxidants or much more?" Cellular and Molecular Life Sciences, vol. 64, no. 22, pp. 2900-2916, 2007.

[8] A. Sahebkar, "A systematic review and meta-analysis of randomized controlled trials investigating the effects of curcumin on blood lipid levels," Clinical Nutrition, 2013.

[9] B. Halliwell, "Oxidative stress and cancer: have we moved forward?” Biochemical Journal, vol. 401, no. 1, pp. 1-11, 2007.

[10] J. L. Marnewick, W. Batenburg, P. Swart, E. Joubert, S. Swanevelder, and W. C. A. Gelderblom, "Ex vivo modulation of chemical-induced mutagenesis by subcellular liver fractions of rats treated with rooibos (Aspalathus linearis) tea, honeybush (Cyclopia intermedia) tea, as well as green and black (Camellia sinensis) teas," Mutation Research, vol. 558, no. 1-2, pp. 145-154, 2004.

[11] L. A. Videla, R. Rodrigo, M. Orellana et al., "Oxidative stressrelated parameters in the liver of non-alcoholic fatty liver disease patients," Clinical Science, vol. 106, no. 3, pp. 261-268, 2004.

[12] B. Uttara, A. V. Singh, P. Zamboni, and R. T. Mahajan, "Oxidative stress and neurodegenerative diseases: a review of upstream and downstream antioxidant therapeutic options," Current Neuropharmacology, vol. 7, no. 1, pp. 65-74, 2009. 
[13] D. L. McKay and J. B. Blumberg, "A review of the bioactivity of South African herbal teas: rooibos (Aspalathus linearis) and honeybush (Cyclopia intermedia)," Phytotherapy Research, vol. 21, no. 1, pp. 1-16, 2007.

[14] C. van Niekerk and A. Viljoen, "Indigenous South African medicinal plants-part 11: Aspalathus linearis ("rooibos")," $S A$ Pharmaceutical Journal, vol. 75, no. 10, pp. 41-42, 2008.

[15] J. F. Morton, "Rooibos tea, Aspalathus linearis, a caffeineless, low-tannin beverage," Economic Botany, vol. 2, pp. 164-173, 1983.

[16] E. Joubert, W. C. A. Gelderblom, A. Louw, and D. de Beer, "South African herbal teas: Aspalathus linearis, Cyclopia spp. and Athrixia phylicoides-a review," Journal of Ethnopharmacology, vol. 119, no. 3, pp. 376-412, 2008.

[17] R. Fukasawa, A. Kanda, and S. Hara, "Anti-oxidative effects of rooibos tea extract on autoxidation and thermal oxidation of lipids," Journal of Oleo Science, vol. 58, no. 6, pp. 275-283, 2009.

[18] J. L. Marnewick, F. H. van der Westhuizen, E. Joubert, S. Swanevelder, P. Swart, and W. C. A. Gelderblom, "Chemoprotective properties of rooibos (Aspalathus linearis), honeybush (Cyclopia intermedia) herbal and green and black (Camellia sinensis) teas against cancer promotion induced by fumonisin $\mathrm{B}_{1}$ in rat liver," Food and Chemical Toxicology, vol. 47, no. 1, pp. 220-229, 2009.

[19] J. Marnewick, E. Joubert, S. Joseph, S. Swanevelder, P. Swart, and W. Gelderblom, "Inhibition of tumour promotion in mouse skin by extracts of rooibos (Aspalathus linearis) and honeybush (Cyclopia intermedia), unique South African herbal teas," Cancer Letters, vol. 224, no. 2, pp. 193-202, 2005.

[20] J. L. Marnewick, F. Rautenbach, I. Venter et al., "Effects of rooibos (Aspalathus linearis) on oxidative stress and biochemical parameters in adults at risk for cardiovascular disease," Journal of Ethnopharmacology, vol. 133, no. 1, pp. 46-52, 2011.

[21] W. G. Pantsi, J. L. Marnewick, A. J. Esterhuyse, F. Rautenbach, and J. van Rooyen, "Rooibos (Aspalathus linearis) offers cardiac protection against ischaemia/reperfusion in the isolated perfused rat heart," Phytomedicine, vol. 18, no. 14, pp. 1220-1228, 2011.

[22] D. O. Awoniyi, Y. G. Aboua, J. L. Marnewick, S. S. du Plesis, and N. L. Brooks, "Protective effects of rooibos (Aspalathus linearis), green tea (Camellia sinensis) and commercial supplements on testicular tissue of oxidative stressinduced rats," African Journal of Biotechnology, vol. 10, no. 75, pp. 17317-17322, 2011.

[23] E. Niki, N. Noguchi, H. Tsuchihashi, and N. Gotoh, "Interaction among vitamin $\mathrm{C}$, vitamin $\mathrm{E}$, and $\beta$-carotene," The American Journal of Clinical Nutrition, vol. 62, supplement 6, pp. 1322S1326S, 1995.

[24] Y. Kadoma, M. Ishihara, and S. Fujisawa, "A quantitative approach to the free radical interaction between alphatocopherol and the coantioxidants eugenol, resveratrol or ascorbate," In Vivo, vol. 20, no. 1, pp. 61-67, 2006.

[25] G. C. M. Bakker, M. J. van Erk, L. Pellis et al., "An antiinflammatory dietary mix modulates inflammation and oxidative and metabolic stress in overweight men: a nutrigenomics approach," The American Journal of Clinical Nutrition, vol. 91, no. 4, pp. 1044-1059, 2010.

[26] Z. Drahota, P. Křiváková, Z. Červinková et al., “Tert-butyl hydroperoxide selectively inhibits mitochondrial respiratorychain enzymes in isolated rat hepatocytes," Physiological Research, vol. 54, no. 1, pp. 67-72, 2005.

[27] S. Singh, S. Mehrotra, R. Pandey, and R. Sandhir, "Hepatotoxic effects of tert-butyl hydroperoxide ( $t$-BHP) and protection by antioxidants," Indian Journal of Experimental Biology, vol. 43, no. 8, pp. 728-731, 2005.

[28] J. M. Oh, Y. S. Jung, B. S. Jeon et al., "Evaluation of hepatotoxicity and oxidative stress in rats treated with tert-butyl hydroperoxide," Food and Chemical Toxicology, vol. 50, no. 5, pp. 1215-1221, 2012.

[29] L. Sissing, J. Marnewick, M. de Kock, S. Swanevelder, E. Joubert, and W. Gelderblom, "Modulating effects of rooibos and honeybush herbal teas on the development of esophageal papillomas in rats," Nutrition and Cancer, vol. 63, no. 4, pp. 600610, 2011.

[30] T. R. Kumar and M. Muralidhara, "Oxidative stress response of rat testis to model prooxidants in vitro and its modulation," Toxicology in Vitro, vol. 16, no. 6, pp. 675-682, 2002.

[31] J.-M. Hwang, C.-J. Wang, F.-P. Chou et al., "Inhibitory effect of berberine on tert-butyl hydroperoxide-induced oxidative damage in rat liver," Archives of Toxicology, vol. 76, no. 11, pp. 664-670, 2002.

[32] A. L. Waterhouse, "Determination of total polyphenolic," Current Protocols in Food Analytical Chemistry, vol. 6, pp. 11.1.111.1.8, 2002.

[33] D. Treutter, W. Feurcht, and C. Santos-Buelga, "Determination of catechins and procyanidins in plant extracts-a comparison of methods," in International Symposium on Natural Phenols in Plant Resistance, vol. 381, pp. 789-796, International Society for Horticultural Science, Weihenstephan, Germany, 1993.

[34] G. Mazza, L. Fukumoto, P. Delaquis, B. Girard, and B. Ewert, "Anthocyanins, phenolics, and color of Cabernet Franc, Merlot, and Pinot Noir wines from British Columbia," Journal of Agricultural and Food Chemistry, vol. 47, no. 10, pp. 4009-4017, 1999.

[35] I. F. F. Benzie and J. J. Strain, "The ferric reducing ability of plasma (FRAP) as a measure of "antioxidant power": the FRAP assay," Analytical Biochemistry, vol. 239, no. 1, pp. 70-76, 1996.

[36] R. Re, N. Pellegrini, A. Proteggente, A. Pannala, M. Yang, and C. Rice-Evans, "Antioxidant activity applying an improved ABTS radical cation decolorization assay," Free Radical Biology and Medicine, vol. 26, no. 9-10, pp. 1231-1237, 1999.

[37] B. Ou, M. Hampsch-Woodill, and R. L. Prior, "Development and validation of an improved oxygen radical absorbance capacity assay using fluorescein as the fluorescent probe," Journal of Agricultural and Food Chemistry, vol. 49, no. 10, pp. 4619-4626, 2001.

[38] R. O. Recknagel and E. A. Glende Jr., "Spectrophotometric detection of lipid conjugated dienes," Methods in Enzymology, vol. 105, pp. 331-337, 1984.

[39] L. M. Ellerby and D. E. Bredesen, "Measurement of cellular oxidation, reactive oxygen species, and antioxidant enzymes during apoptosis," Methods in Enzymology, vol. 322, pp. 413-421, 2000.

[40] L. Flohé and W. A. Günzler, "Assays of glutathione peroxidase," Methods in Enzymology, vol. 105, pp. 114-121, 1984.

[41] P. C. H. Hollman, "Absorption, bioavailability, and metabolism of flavonoids," Pharmaceutical Biology, vol. 42, pp. 74-83, 2004.

[42] S. Kreuz, E. Joubert, K.-H. Waldmann, and W. Ternes, "Aspalathin, a flavonoid in Aspalathus linearis (rooibos), is absorbed by pig intestine as a C-glycoside," Nutrition Research, vol. 28, no. 10, pp. 690-701, 2008.

[43] G.-C. Yen, C.-T. Yeh, and Y.-J. Chen, "Protective effect of Mesona procumbens against tert-butyl hydroperoxide-induced acute hepatic damage in rats," Journal of Agricultural and Food Chemistry, vol. 52, no. 13, pp. 4121-4127, 2004. 
[44] L. J. Roberts II and J. D. Morrow, "Products of the isoprostane pathway: unique bioactive compounds and markers of lipid peroxidation," Cellular and Molecular Life Sciences, vol. 59, no. 5, pp. 808-820, 2002.

[45] M.-H. Khadem-Ansari, Z. Shahsavari, Y. Rasmi, and R. Mahmoodlo, "Elevated levels of urinary 8-hydroxy-2' deoxyguanosine and 8-isoprostane in esophageal squamous cell carcinoma," Journal of Carcinogenesis, vol. 10, article 14, 2011.

[46] S. S. Davies and L. J. Roberts II, "F $F_{2}$-isoprostanes as an indicator and risk factor for coronary heart disease," Free Radical Biology and Medicine, vol. 50, no. 5, pp. 559-566, 2011.

[47] J. L. Marnewick, "Rooibos and honeybush: recent advances in chemistry, biological activity and pharmacognosy," in African Natural Plant Products: New Discoveries and Challenges in Chemistry and Quality, H. R. Juliana, J. E. Simon, and C.-T. Ho, Eds., vol. 1021 of ACS Symposium Series, pp. 277-294, Oxford University Press, Oxford, UK, 2009.

[48] P. Pedrielli and L. H. Skibsted, "Antioxidant synergy and regeneration effect of quercetin, (-)-epicatechin, and (+)-catechin on $\alpha$-tocopherol in homogeneous solutions of peroxidating methyl linoleate," Journal of Agricultural and Food Chemistry, vol. 50, no. 24, pp. 7138-7144, 2002.

[49] A. Bendich, P. D’Apolito, E. Gabriel, and L. J. Machlin, "Interaction of dietary vitamin $\mathrm{C}$ and vitamin $\mathrm{E}$ on guinea pig immune responses to mitogens," The Journal of Nutrition, vol. 114, no. 9, pp. 1588-1593, 1984.

[50] D. P. Jain, S. S. Pancholi, and R. Patel, "Synergistic antioxidant activity of green tea with some herbs," Journal of Advanced Pharmaceutical Technology \& Research, vol. 2, no. 3, pp. 177-183, 2011.

[51] A. Karadag, B. Ozcelik, and S. Saner, "Review of methods to determine antioxidant capacities," Food Analytical Methods, vol. 2, no. 1, pp. 41-60, 2009.

[52] S. B. Lotito and B. Frei, "Consumption of flavonoid-rich foods and increased plasma antioxidant capacity in humans: cause, consequence, or epiphenomenon?" Free Radical Biology and Medicine, vol. 41, no. 12, pp. 1727-1746, 2006.

[53] H. R. Griffiths, L. Møller, G. Bartosz et al., "Biomarkers," Molecular Aspects of Medicine, vol. 23, no. 1-3, pp. 101-208, 2002.

[54] A. R. Collins, "Assays for oxidative stress and antioxidant status: applications to research into the biological effectiveness of polyphenols," The American Journal of Clinical Nutrition, vol. 81, supplement 1, pp. 261S-267S, 2005.

[55] V. Dilis and A. Trichopoulou, "Antioxidant intakes and food sources in Greek adults," The Journal of Nutrition, vol. 140, no. 7, pp. 1274-1279, 2010.

[56] D. Villaño, M. Pecorari, M. F. Testa et al., "Unfermented and fermented rooibos teas (Aspalathus linearis) increase plasma total antioxidant capacity in healthy humans," Food Chemistry, vol. 123, no. 3, pp. 679-683, 2010.

[57] A. Stalmach, W. Mullen, M. Pecorari, M. Serafini, and A. Crozier, "Bioavailability of C-linked dihydrochalcone and flavanone glucosides in humans following ingestion of unfermented and fermented rooibos teas," Journal of Agricultural and Food Chemistry, vol. 57, no. 15, pp. 7104-7111, 2009.

[58] B. Halliwell, "The wanderings of a free radical," Free Radical Biology and Medicine, vol. 46, no. 5, pp. 531-542, 2009.

[59] K. Hamden, S. Carreau, F. Ellouz, H. Masmoudi, and A. El Feki, "Improvement effect of green tea on hepatic dysfunction, lipid peroxidation and antioxidant defence depletion induced by cadmium," African Journal of Biotechnology, vol. 8, no. 17, pp. 4233-4238, 2009.

[60] V. G. Raneva and H. Shimasaki, "Green tea catechins decrease lipid peroxidation in plasma and organs of C57BL/6J mice fed atherogenic diet," Journal of Oleo Science, vol. 54, no. 12, pp. 641648, 2005.

[61] O. Uličná, O. Vančová, P. Božek et al., "Rooibos tea (Aspalathus linearis) partially prevents oxidative stress in streptozotocininduced diabetic rats," Physiological Research, vol. 55, no. 2, pp. 157-164, 2006.

[62] J. M. Hodgson, K. D. Croft, T. A. Mori, V. Burke, L. J. Beilin, and I. B. Puddey, "Regular ingestion of tea does not inhibit in vivo lipid peroxidation in humans," The Journal of Nutrition, vol. 132, no. 1, pp. 55-58, 2002.

[63] B. Halliwell, "Dietary polyphenols: good, bad, or indifferent for your health?" Cardiovascular Research, vol. 73, no. 2, pp. 341$347,2007$.

[64] A. Aguiló, P. Tauler, E. Fuentespina, J. A. Tur, A. Córdova, and A. Pons, "Antioxidant response to oxidative stress induced by exhaustive exercise," Physiology \& Behavior, vol. 84, no. 1, pp. $1-7,2005$

[65] S. Dudonné, X. Vitrac, P. Coutiére, M. Woillez, and J.-M. Mérillon, "Comparative study of antioxidant properties and total phenolic content of 30 plant extracts of industrial interest using DPPH, ABTS, FRAP, SOD, and ORAC assays," Journal of Agricultural and Food Chemistry, vol. 57, no. 5, pp. 1768-1774, 2009. 


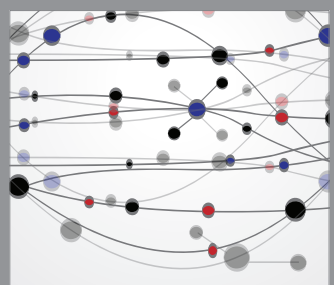

The Scientific World Journal
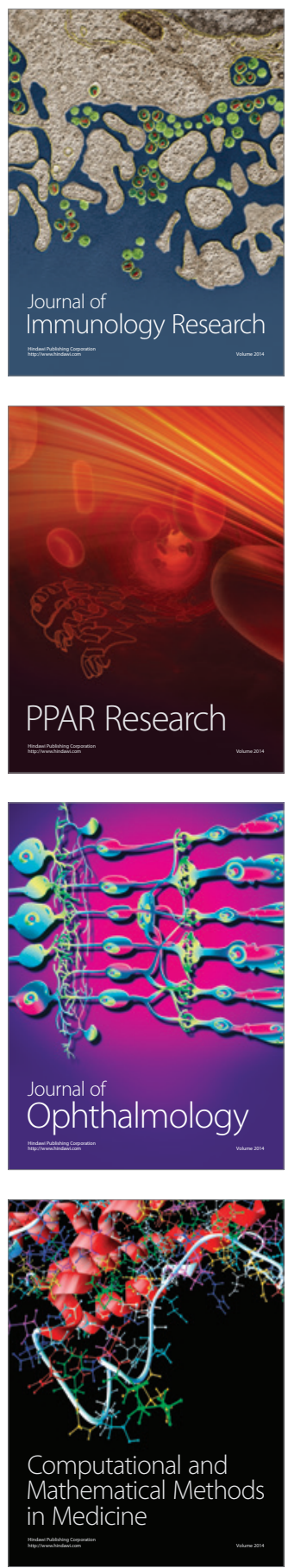

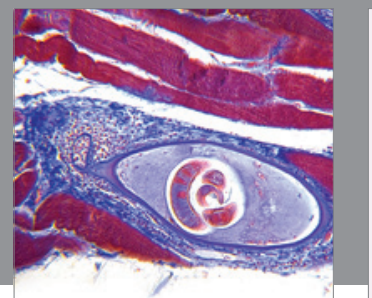

Gastroenterology

Research and Practice
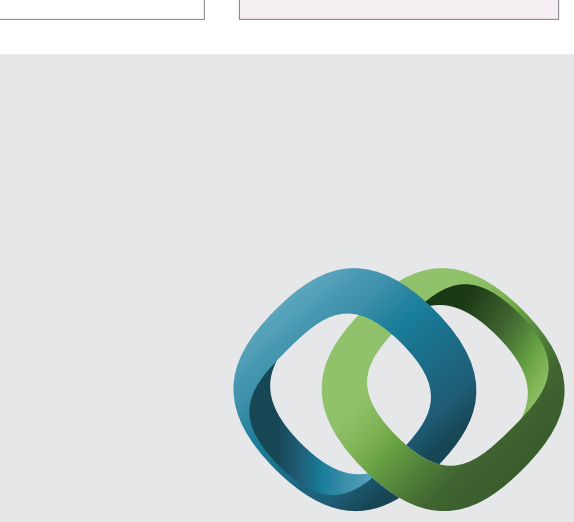

\section{Hindawi}

Submit your manuscripts at

http://www.hindawi.com
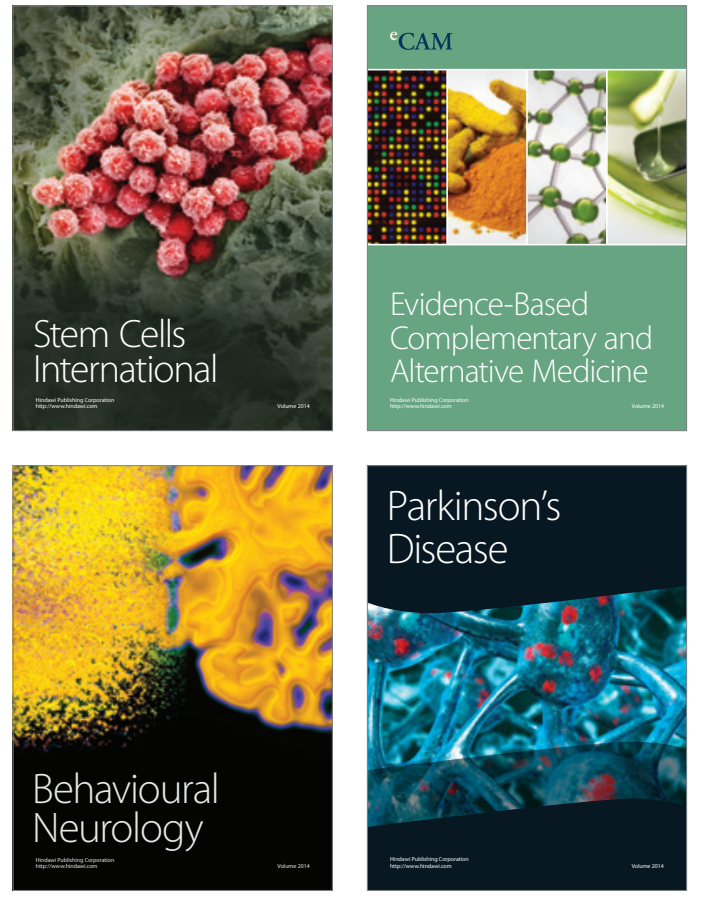
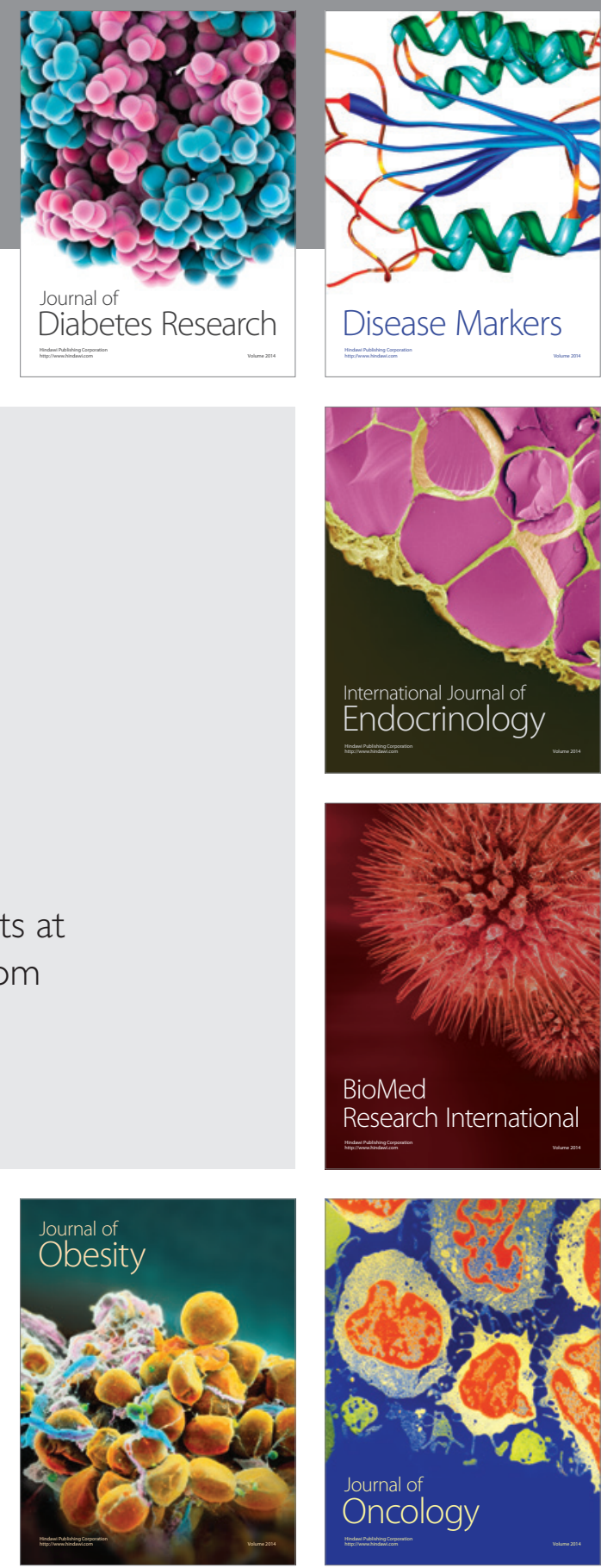

Disease Markers
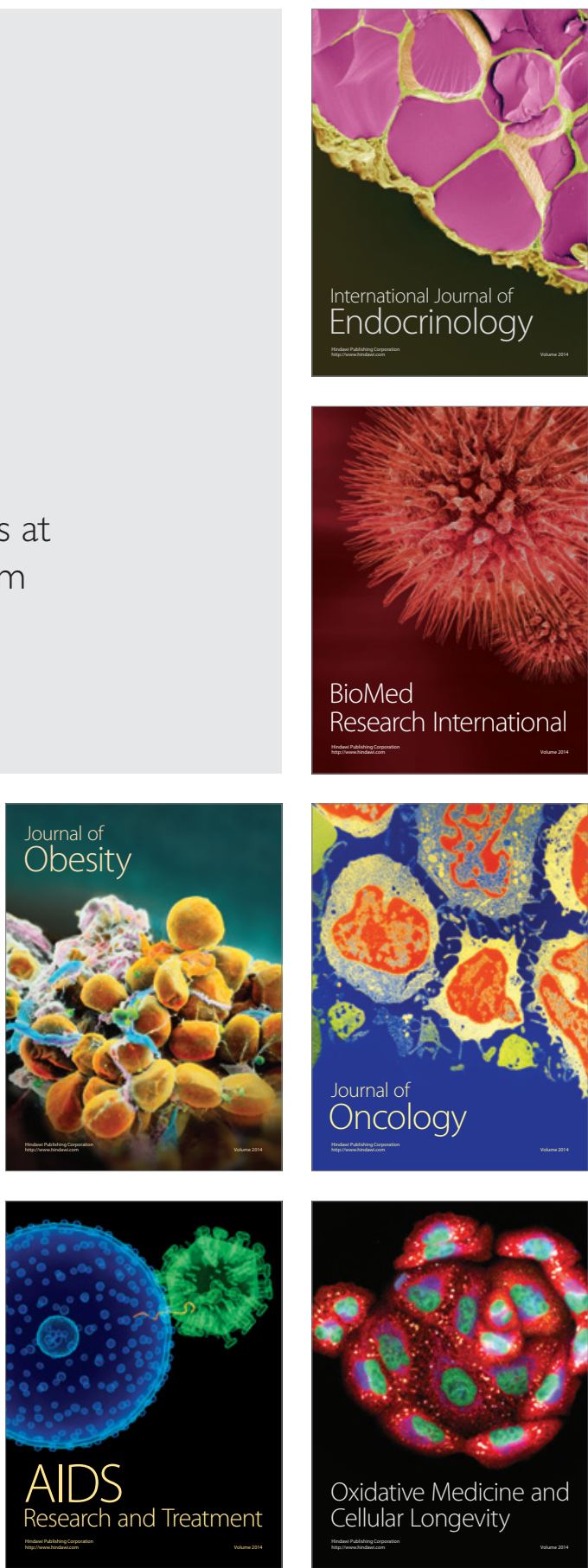